The Chittagong Univ. J. Sci. 43(1): 41-50, 2021

\title{
Variational Analysis of the Speed of Propagating Fronts in Superconductors
}

\author{
Neelufar Panna \\ Department of Physics, University of Chittagong, Chittagong-4331, Bangladesh \\ E-mail: neelcu@yahoo.com
}

Manuscript Submitted on 01/03/2021, Revised manuscript received on 04/04/2021, and accepted on $06 / 05 / 2021$

\begin{abstract}
A new approach has taken to determine the speed of the superconducting-normal interface (front) propagating from a stable superconducting phase to the unstable normal phase by means of a variational method. In this process a trial function $\mathrm{g}(\mathrm{x})$ is introduced which has a relation with the superconducting order parameter $F$. Starting from the time-dependent Ginzburg-Landau (TDGL) equations an expression is derived for the front speed in terms of GL parameter $\kappa$.
\end{abstract}

Keywords: Front propagation in superconductor; Ginzburg-Landau equation; velocity selection; exact solution; variational analysis.

DOI: https://doi.org/10.3329/cujs.v43i1.57333

যখন অতিপরিবাহী-স্বাভাবিক একটি অন্তঃপৃষ্ঠ অতিপরিবাহীর স্থায়ী দশা থেকে স্বাভাবিক বিশৃংখল দশায় প্রবেশ করে ভেরিয়েশনাল পদ্ধতিতে তখন অতিপরিবাহীতে আন্তঃপৃষ্ঠবিস্তারণের দ্রুতি নির্ণয় করা হয়েছে। এ পদ্ধতিতে অতিপরিবাহীর অর্ডার প্যারামিটার $F$ এর সাথে সম্পর্কযুক্ত একটি ট্রায়াল ফাংশান $\mathrm{g}(\mathrm{x})$ অর্ত্তভুক্ত করা হয়েছে। সময় নির্ভর Ginzburg-Landau (TDGL) সমীকরণ ব্যবহার করে আন্তঃপৃষ্ঠবিস্তারণের দ্রততির জন্য একটি সমীকরণ নির্ণীত হয়েছে যা G-L প্যারামিটার $\kappa$ এর উপর নির্ভরশীল।

\section{Introduction}

In the last few years the propagation of fronts into an unstable state gives several dynamical problems. Such fronts arise in diverse fields as biology, population dynamics, pulse propagation in nerves, fluid flow, liquid crystals and the propagation of a superconducting front into a normal metal. There have been several attempts to calculate the front speed such as the marginal stability hypothesis (MSH) [1], construction of exact solutions [2], variational method [3] etc. 
According to the work of $\mathrm{Di}$ Bartolo and Dorsey [1] consider a sample of superconducting material placed in a stationary applied magnetic field $\mathrm{H}$ equal to the critical field Hc so that there is a stationary planar superconducting-normal interface grows which separates the normal and superconducting phases. After than the magnetic field is rapidly removed, the interface becomes dynamically unstable and propagates towards the normal phase so as to expel any trapped magnetic flux, leaving the sample in Meissner State.

Bartolo and Dorsey calculate the speed of this front using MSH. We have obtained the front speed by exact solutions $[4,5]$. The variational characterization for the speed of the fronts of the nonlinear diffusion equation is discussed. Fisher's nonlinear diffusion equation is $[6,7]$

$u_{t}=u_{x x}+F(u)$

where $u>0$ is referred to as a population density and $F(0)=F(1)=0 . \quad F$ is positive in $(0,1)$. In this case $u=0$ is the unstable fixed point and $u=1$ is a stable fixed point. Aronson and of the Weinberger [8] have shown that for a sufficiently localized initial condition the solution of this equation evolve into a front that join the stable state $u=1$ to unstable state $u=0$ with a definite speed. Moreover they show that the selected speed is bounded above and below by

$2 \sqrt{F^{\prime}(0)} \leq v \leq 2 \sup \sqrt{F(u) / u}$

An important problem to be solved is the determination of the speed at which the interface moves from a superconducting to a normal state. The aim of this paper is to determine the speed of the propagating front in a superconducting sample from a variational point of view. The paper is organized as follows. In section 2 the front speed has discussed in terms of GL parameter $\kappa$. Section 3 provides the results and discussions. 


\section{Theory}

The time-dependent Ginzberg-Landau (TDGL) equations are the starting points for the study of the dynamics of the front propagation in superconductors. These equations provide the self-consistent description of the coupling between the order parameter and the vector potential.

One dimensional TDGL equations in dimensionless unit are introduced as:

$$
\partial_{t} f=\frac{1}{\kappa^{2}} \partial_{\mathrm{x}}^{2} f-q^{2} f+f-f^{3}
$$

$\bar{\sigma} \partial_{t} q=\partial_{x}^{2} q-f^{2} q$

Here $\mathrm{f}$ is the magnitude of the superconducting order parameter, $\mathrm{q}$ is the vector potential which is gauge invariant (such that $h=\partial_{x} q$ is the magnetic field) $\bar{\sigma}$ is the dimensionless normal state conductivity and $\kappa$ is the Ginzberg-Landau parameter.

To begin our variational analysis it is important to use the steady monotonic traveling front of the TDGL eqs. which are of the form $f(x, t)=F(X)=F(x-v t)$ and $q(x, t)=Q(X)=Q(x-v t)$, where $X=x-v t$ with $v>0$.

Then the TDGL eqs. become

$$
\begin{aligned}
& \frac{1}{\kappa^{2}} F_{x x}+v F_{x}-Q^{2} F+F-F^{3}=0 \\
& Q_{x x}+\bar{\sigma} v Q_{x}-F^{2} Q=0
\end{aligned}
$$

For variational study let $q=0$ for GL eq. (1)

$$
\partial_{t} f=\frac{1}{\kappa^{2}} \partial_{\mathrm{x}}^{2} f+f-f^{3}
$$


44 Neelufar Panna

This results a front $f=F(x-v t)$ which joins the state corresponding to the superconducting phase $f=1$ to the normal state $f=0$. The two states are connected with speed $v$ by traveling wave front.

With the boundary conditions

$\lim _{x \rightarrow-\infty} f=1$ and $\lim _{x \rightarrow \infty} f=0$

Eq. (5) can be written as

$F_{x x}+v \kappa^{2} F_{x}+\kappa^{2}\left(F-F^{3}\right)=0 \quad$ for $Q=0$

$F_{x x}+v \kappa^{2} F_{x}+\mu_{\kappa}(F)=0$

where $\mu_{\kappa}(F)=\kappa^{2} F\left(1-F^{2}\right)$ and $\mu=\frac{1}{\kappa^{2}} \mu_{\kappa}(F)$

Since the selected speed corresponds to that of a decreasing monotonic front, the dependence of its derivative $\frac{d F}{d X}$ on $F$ is defined as [9]

$p(F)=-\frac{d F}{d X}=-F_{x}$

The negative sign is included so that $p$ is positive.

$\frac{d p}{d F}=\frac{d}{d F}\left(-\frac{d F}{d X}\right)=-\frac{d}{d X}$

$\therefore p(F) \frac{d p}{d F}=-\frac{d F}{d X}\left(-\frac{d}{d X}\right)=\frac{d^{2} F}{d X^{2}}=F_{x x}$

Then the monotonic fronts, eq. (6) become the solution of

$p(F) \frac{d p}{d F}-v \kappa^{2} p(F)+\mu_{\kappa}(F)=0$

with $p(0)=0, p(1)=0, p>0$ in $(0,1)$.

Benguria and Depassier proposed a trial function $g(x)$ which satisfy the conditions $g(F)>0$ and $g^{\prime}(F)<0$ in $(0,1)$ such that $h=-\frac{d g}{d F}>0$. 
Multiplying eq. (7) by $g(F)$ and integrating with respect to $F$ and after simplification eq. (7) can be written as

$$
\int_{0}^{1}\left(h p+\frac{\mu_{\kappa}(F)}{p} g\right) d F=v \int_{0}^{1} g d F
$$

Let us take $h p+\left(\frac{\mu_{\kappa}(F)}{p} g\right) \geq 2 \sqrt{g h \mu_{\kappa}}$,

then the result for speed is

$$
v=\frac{2}{\kappa} \frac{\int_{0}^{1}(g h \mu)^{1 / 2} d F}{\int_{0}^{1} g d F}
$$

In this paper we choose the trial function as

$$
g(F)=1-F
$$

Where the order parameter $F(X)$ is given by [10]

$F(X)=\frac{1}{1+\alpha e^{\xi X}}$

Now eq. (8) has the form with eq. (9) and by using $h=-\frac{d g}{d F}$ as 
$v \geq \frac{2}{\kappa} \frac{\int_{0}^{1}\left[(1-F) F\left(1-F^{2}\right)\right]^{1 / 2}}{\int_{0}^{1}(1-F) d F} d F$

Substituting the value of $F$ from eq. (10) into eq. (11), we get

$$
\begin{aligned}
& v \geq \frac{2}{\kappa} \frac{\int_{0}^{1}\left[\frac{1}{1+\alpha e^{\xi X}}\left(1-\frac{1}{1+\alpha e^{\xi X}}\right)\left\{1-\left(\frac{1}{1+\alpha e^{\xi X}}\right)^{2}\right\}\right]^{\frac{1}{2}} d X}{\int_{0}^{1}\left(1-\frac{1}{1+\alpha e^{\xi X}}\right) d X} \\
& v \geq \frac{2}{\kappa} \frac{\int_{0}^{1}\left[\frac{\left(2 \alpha^{2} e^{2 \xi X}+\alpha^{3} e^{3 \xi X}\right)}{\left(1+\alpha e^{\xi X}\right)^{4}}\right]^{\frac{1}{2}} d X}{\int_{0}^{1}\left(\frac{\alpha e^{\xi X}}{1+\alpha e^{\xi X}}\right) d X}
\end{aligned}
$$

For $\alpha=1$ and $\xi=\frac{1}{\sqrt{2}}$

$$
v \geq \frac{2}{\kappa} \frac{\int_{0}^{1}\left[\left(2 e^{\sqrt{2} X}+e^{3 X / \sqrt{2}}\right)^{\frac{1}{2}} /\left(1+e^{X / \sqrt{2}}\right)^{2}\right] d X}{\int_{0}^{1}\left[e^{X / \sqrt{2}} /\left(1+e^{X / \sqrt{2}}\right)\right] d X}
$$


$v \geq \frac{2}{\kappa} \frac{\sqrt{2} \int_{0}^{1}\left[\left\{e^{\sqrt{2} X}\left(1+\frac{1}{2} e^{X / \sqrt{2}}\right)\right\}^{\frac{1}{2}}\left\{\left(1+e^{X / \sqrt{2}}\right)^{-2}\right\} d X\right.}{\int_{0}^{1} e^{X / \sqrt{2}}\left(1+e^{X / \sqrt{2}}\right)^{-1} d X}$

$v \geq \frac{2 \sqrt{2}}{\kappa} \frac{\int_{0}^{1} e^{\sqrt{2} X}\left[\left\{1+\left(\frac{1}{2}\right)\left(\frac{1}{2}\right) e^{X / \sqrt{2}}\right\}\left\{\left(1-2 e^{X / \sqrt{2}}\right)\right\}\right] d X}{\int_{0}^{1} e^{X / \sqrt{2}}\left(1-e^{X / \sqrt{2}}\right) d X}$

$v \geq \frac{2 \sqrt{2}}{\kappa} \frac{\int_{0}^{1}\left[e^{\sqrt{2} X}+\frac{1}{4} e^{3 X / \sqrt{2}}-2 e^{3 X / \sqrt{2}}-\frac{1}{2} e^{4 X / \sqrt{2}}\right] d X}{\int_{0}^{1}\left(e^{X / \sqrt{2}}-e^{\sqrt{2} X}\right) d X}$

$v \geq \frac{2 \sqrt{2}}{\kappa} \frac{\left[e^{\sqrt{2}}-1.166 e^{3 / \sqrt{2}}-\frac{1}{4} e^{4 / \sqrt{2}}+\frac{5}{12}\right]}{\left[\sqrt{2} e^{1 / \sqrt{2}}-\frac{1}{\sqrt{2}} e^{\sqrt{2}}-\frac{1}{\sqrt{2}}\right]}$

$v \geq \frac{25.2}{\kappa}$

This is the front speed in terms of GL parameter. 


\section{Results and Discussion}

In this paper the variational criteria has derived to study the dependence of the front speed on the GL parameter in superconductors. The propagation of interface separating the superconducting and normal phases are produced after a quench to zero applied magnetic field. A trial function $g(F)=1-F$ is used for the order parameter $F(X)=\frac{1}{1+\alpha e^{\xi X}}$.

From eq. (12) the speed in terms of GL parameter for which superconducting interface exists can be obtained as follows:

$v \geq \frac{25.2}{\kappa}$

which means the rate at which the magnetic flux invade into the normal state.

In Figure 1 the front speed vversus the GL parameter $\kappa$ is plotted. The speed has represented from the variational point of view by using a trial function $g$. The graphshows that the front speed tends to Benguria and Depassier value for $\kappa>8$ and for $\kappa<8$ the variational speed selection gives a better lower bound. The result is in good agreement with the result for speed obtained from [11]. 


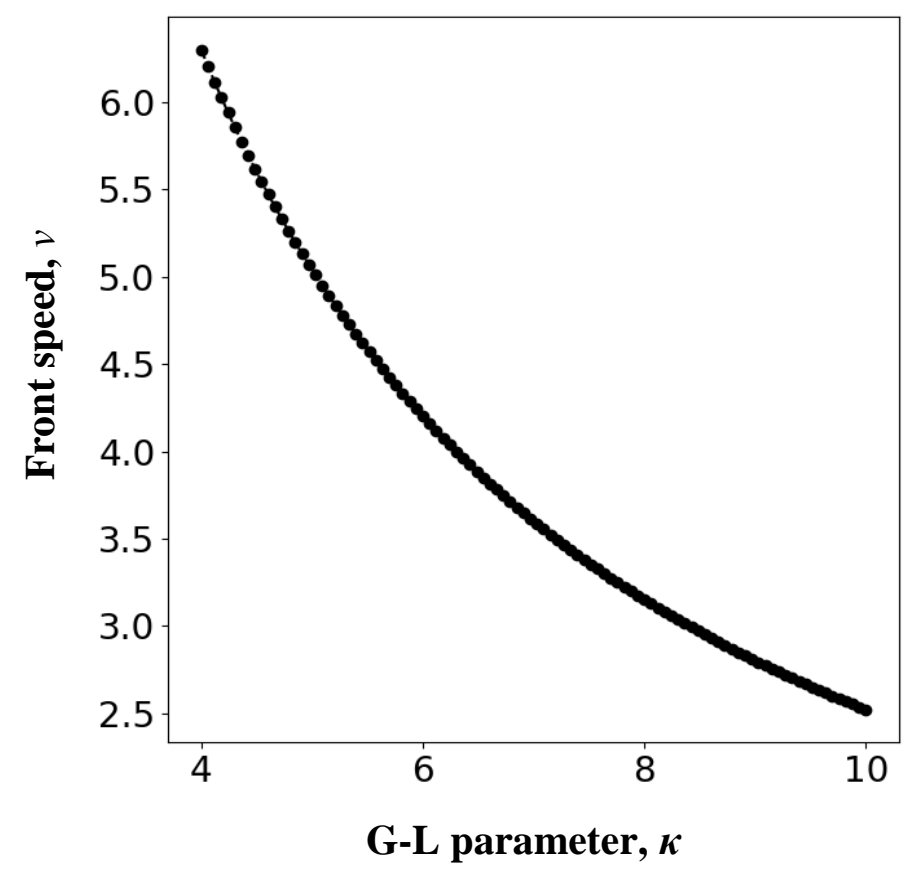

Figure 1. The graph represents the front speed obtained from eq. (12) as a function of G-L parameter $\kappa$.

\section{Conclusion}

The interface propagation in superconductor by means of a variational method is studied. The variational speed characterization or BD method was proposed by Benguria and Depassier in order to determine the reaction diffusion equations. Using a trial function $g(x)$ one may find accurate lower and upper bounds for the speed.In this paper I introduced a trial function in the form $g(F)=1-F$, where $F(X)=\frac{1}{1+\alpha e^{\xi X}}$ is the order parameter in superconductors. In this procedure an expression is derived for the lower bound of the front speed in terms of G-L 
parameter given as $v \geq \frac{25.2}{\kappa}$. In my future work I will try to evaluate the lower and upper bound of superconducting-normal interface speed by parabolic and hyperbolic diffusion equations.

\section{References}

[1] S. J. Di Bartolo and A. T. Dorsey: Phys. Rev. Lett., 1996, 77(21), 4442-4445.

[2] W. van Saarloos: Phys. Rev A, 1989, 39, 6367-6390.

[3] R. D. Benguria and M.C. Depassier: Phys. Rev. Lett., 1994, 73, 2272-2275.

[4] Neelufar Panna and J. N. Islam: Pramana-Indian Academy of Sciences J. Phys., 2013, 80(5), 895-901.

[5] Neelufar Panna: The Chittagong University Journal of Science, 2020. (In Press)

[6] R. A. Fisher: Ann. Eugen., 1937, 7, 355-369.

[7] A. N. Kolmogorov, I. G. Petrovsii and N. S. Piskunov: Bull. Univ.- Moscow, Ser. Int. A, 1937, 1,1-25.

[8] D. G. Aronson and H.F. Weinberge: Adv. Math., 1978, 30, 33-76.

[9] R. D. Benguria and M. C. Depassier: Commun. Math. Phys. 1996, 175, 221-227.

[10] Neelufar Panna: Ph.D. Thesis, 2013.

[11] A.de la Cruz de Ona: cond-mat-supr-con., 2008, eprint, arXiv:0705.0896v1.

The Chittagong Univ. J. Sc. Vol. 43(1), 2021 